\title{
THRESHOLD EFFECT OF CAFFEIC ACID ON PEROXIDASE-CATALYSED OXIDATION OF INDOLE-3-ACETIC ACID
}

\author{
Sergey N. Krylov,* Svetlana M. Krylova and Leonid B. Rubin \\ Institute for Nuclear Physics Research, Moscow State University, GSP, Moscow 119899, Russia
}

(Received in revised form 22 September 1992)

\begin{abstract}
Key Word Index-Horseradish peroxidase; oxidation of indole-3-acetic acid; inhibition; free radical
\end{abstract} chain reaction; caffeic acid.

\begin{abstract}
The hitherto unknown threshold effect for the inhibition of the peroxidase-catalysed aerobic oxidation of indole-3-acetic acid (IAA) by caffeic acid is described. It is shown that caffeic acid does not affect peroxidase and IAA. To explain the threshold effect it is supposed that for the given experimental conditions, the oxidation of IAA is an enzymatic, free radical, chain branched reaction and caffeic acid acts as a trap of free radicals participating in the chain reaction.
\end{abstract}

\section{INTRODUCTION}

Indole-3-acetic acid (IAA) is a natural phytohormone with many growth regulatory functions. Enzymatic oxidation of IAA is catalysed by IAA-oxidases, many of which are peroxidases [1]. Peroxidase-catalysed IAA oxidation is, in turn, affected by natural phenolic inhibitors. The influence of the inhibitors on this reaction was carefully investigated by a number of researchers [2-7]. According to the prevalent opinion, competitive enzyme inhibition is realized $[2,4-6]$. This hypothesis, however, has a number of drawbacks: in particular the Lineweaver-Burk plots are curvilinear $[5,7]$. Here we describe the previously unknown threshold effect for the inhibition of the peroxidase-catalysed oxidation of IAA by caffeic acid (a natural inhibitor of this reaction [3]).

\section{RESULTS}

Studying the influence of caffeic acid on the peroxidasecatalysed oxidation of IAA, we found a previously unknown threshold effect. If the concentration of the inhibitor was less than the threshold value, only temporary decreases of the intensity of chemiluminescence (Fig. 1) and rate of IAA oxidation (Fig. 2) were observed. If the inhibitor concentration was more than the threshold value, then the chemiluminescence ended (Fig. 1), the reaction stopped (Fig. 2) and the process rate was zero during the entire observation time ( $36 \mathrm{hr}$ ). Enzyme addition did not reinitiate the reaction, nor did dilution of the reaction mixture by the buffer influence the stopped reaction. Upon adding the extra substrate solution, the

*Author to whom correspondence should be addressed.

†The new substance under patent pending. Please contact Sergey N. Krylov for additional information. reaction was reinitiated. Moreover, the reaction reinitiation also took place when the reaction mixture was irradiated by visible light in the presence of a photosensitizer-rose bengal. The reaction proceeded without light and had ordinary kinetics after initiation. Irradiation dosage and rose bengal concentration required for reinitiation increased with the inhibitor concentration.

The value of the threshold inhibitor concentration increased with the enzyme and substrate concentrations, only when the inhibitor was added to the substrate after the enzyme (Fig. 3, curve 1). If the inhibitor was added to the substrate before the enzyme, however, then threshold inhibitor concentration did not depend on the enzyme concentration, but was proportional to the substrate concentration (Fig. 3, curve 2). The threshold effect of caffeic acid was observed during the whole reaction time. The threshold concentration of the inhibitor depended on the moment of the inhibitor addition (Fig. 4).

Results similar to those described above were also obtained using anphent (a free radical trap) as the inhibitor. One must emphasize that anphen is not a natural product. The threshold concentration for this substance was about $20 \times$ more than that for caffeic acid.

\section{DISCUSSION}

The failure of enzyme addition to initiate the stopped reaction leads one to the conclusion that caffeic acid does not influence the peroxidase. This conclusion is supported by the fact that threshold inhibitor concentration does not depend on the enzyme concentration when the inhibitor is added to the IAA solution before the enzyme. The threshold inhibitor concentration is about $10^{-3} \times$ the initial substrate concentration. Moreover, the chemiluminescent kinetics of the reaction reinitiated by light 

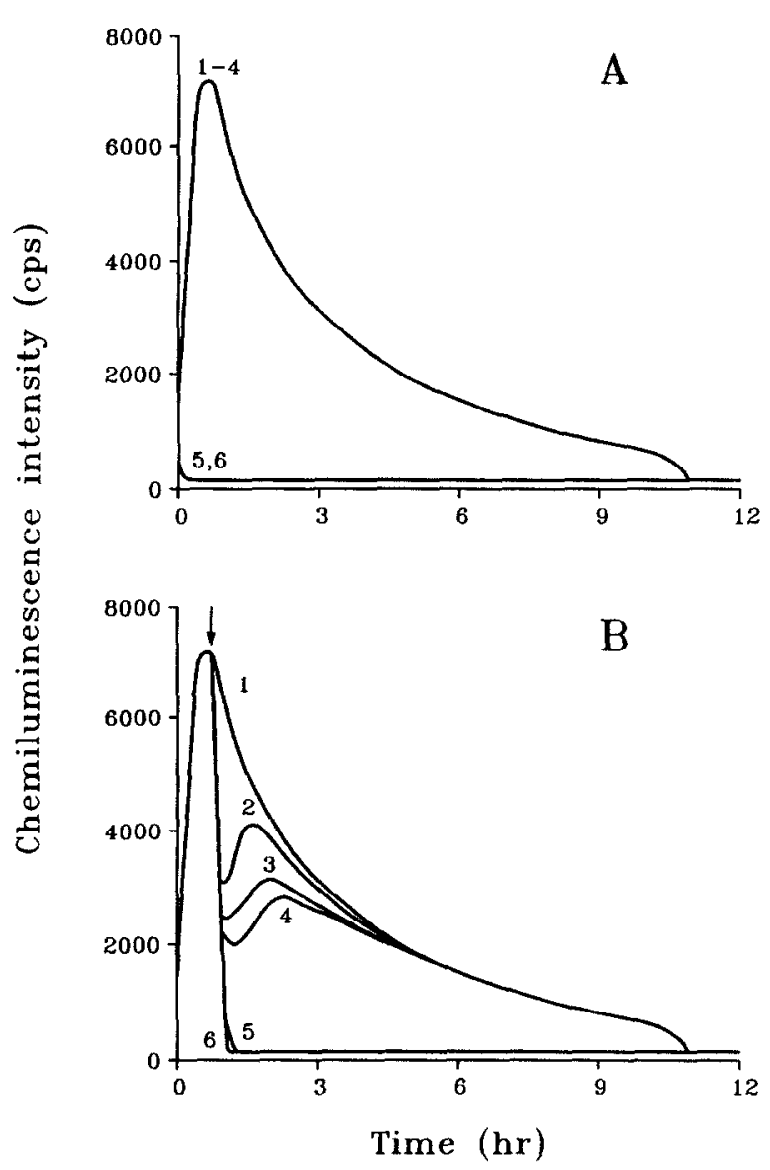

Fig. 1. The effect of caffeic acid on the chemiluminescence kinetics for the IAA (1 mM) oxidation catalysed by peroxidase $(0.1 \mu \mathrm{M})$. (A) Peroxidase was added to IAA solution after caffeic acid; concentration of caffeic acid $(\mu \mathrm{M}): 0$ (1), 0.08 (2), 0.16 (3), 0.24 (4), 0.33 (5), 0.41 (6). (B) Caffeic acid was added to the reaction mixture $45 \mathrm{~min}$ after the reaction initiation (arrow shows the moment of caffeic acid addition); concentration of caffeic acid $(\mu \mathrm{M})$ : $0(1), 1.3(2), 2.3(3), 3.2(4), 4.0(5), 4.9$ (6).

irradiation after the cessation by the inhibitor are identical to those of the reaction subjected to no influences. It follows that the inhibitor does not affect the substrate.

We suppose that caffeic acid acts on some hypothetical substance R. Traces of this substance would be found in the substrate solution. This is confirmed by the fact that the stopped reaction is reinitiated when an extra substrate solution is added. Moreover, $\mathbf{R}$ is likely to be produced during the peroxidase-catalysed IAA oxidation. The foundation for this proposal is the dependence of the threshold inhibitor concentration on the substratc and enzyme concentrations (Fig. 3) and on the moment of inhibitor addition (Fig. 4). The nature of substance $R$ is unknown. However, the following indirect data allow one to suppose that $\mathbf{R}$ is a free radical. The stopped reaction can be reinitiated by light irradiation in the presence of rose bengal. Rose bengal is known to give a high yield of a triplet excited species when it is irradiated by light [10]. The triplet excited species are, per se, biradicals. Their presence can cause intensive free radical formation [11].
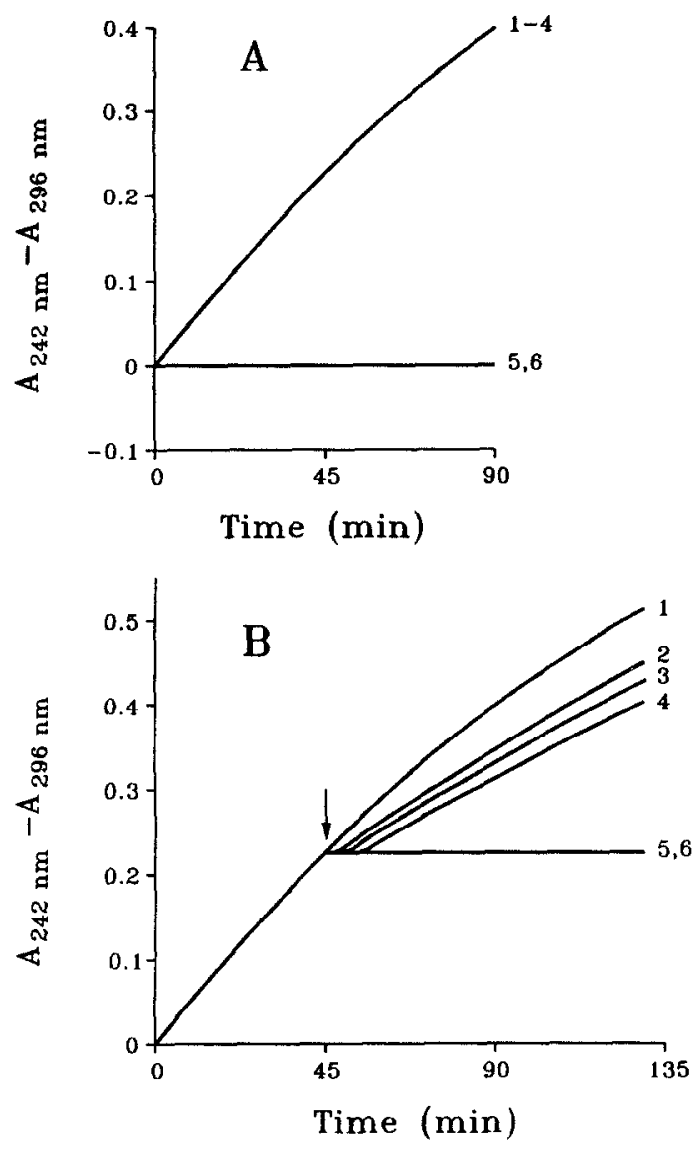

Fig. 2. The effect of caffeic acid on the kinetics of the IAA (1 $\mathrm{mM}$ ) oxidation, catalysed by peroxidase $(0.1 \mu \mathrm{M})$. A and $\mathrm{B}$ are similar to Fig. 1.

Moreover, it is known that free radicals are formed during peroxidase-catalysed IAA oxidation. In particular, the Skatole-radical was identified [12]. If this hypothcsis of the free-radical nature of substance $R$ is valid, then caffeic acid should act as a free radical trap. The confirmation of this proposal is the fact that the effect of anphen (a free radical trap) on the peroxidase-catalysed IAA oxidation is similar to that of caffeic acid.

Therefore, in order to explain the threshold effect, we suppose that IAA oxidation is an enzymatic, free radical, chain branched reaction and caffeic acid quenches the free radicals participating in the chain reaction by means of a free radical transfer process:

$$
\mathbf{R}^{\cdot}+\mathbf{I} \rightarrow \mathbf{R}+\mathbf{I}^{\bullet}
$$

Frec radicals of inhibitor $\mathbf{I}$, which are formed in this case, are quickly dissipated, due to the formation of dimers:

$$
\mathbf{I}^{\cdot}+\mathbf{I} \rightarrow \mathbf{I}-\mathbf{I}
$$

If the inhibitor concentration is more than the threshold value, then the concentration of free radicals $R^{*}$ decreases such that the free radical multiplication factor is less than one and the chain reaction stops. If the inhibitor concentration is less than the threshold value, then the free radical multiplication factor is more than one and the 


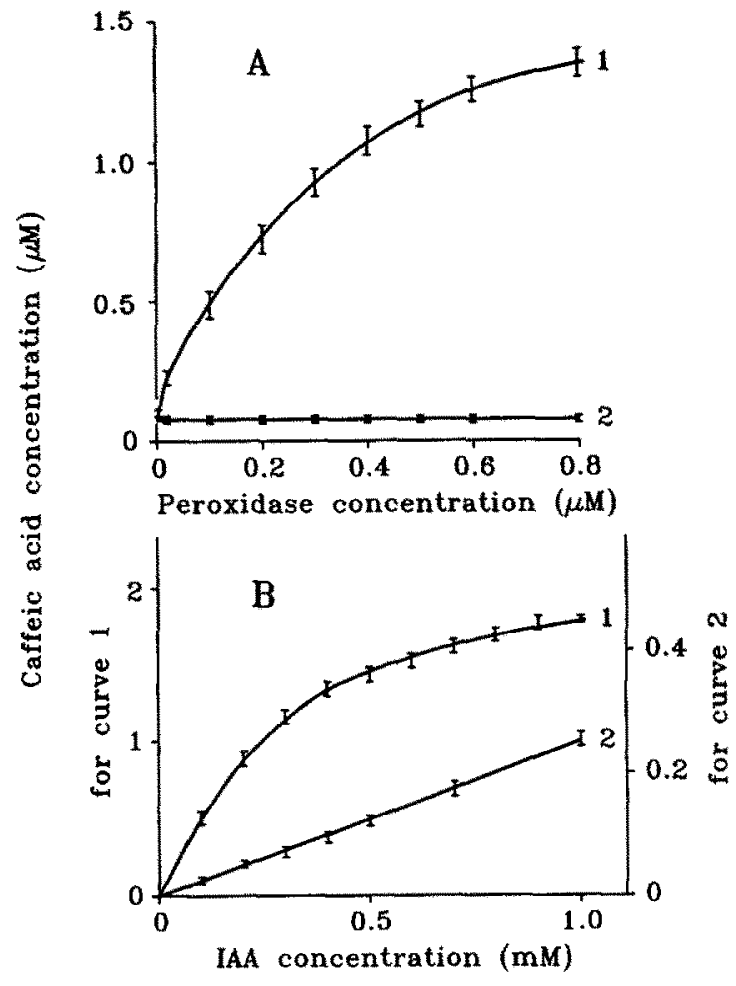

Fig. 3. The effect of peroxidase (A) and IAA (B) concentrations on the minimal caffeic acid concentration required for the cessation of the peroxidase-catalysed IAA oxidation. (1) Caffeic acid was added $3 \mathrm{~min}$ after IAA-peroxidase mixture preparation. (2) Caffeic acid was added to the IAA solution before the peroxidase addition. (A) IAA concentration is $0.1 \mathrm{mM},(B)$ peroxidase concentration is $0.1 \mu \mathrm{M}$.

chain reaction develops as an avalanche, increasing the concentration of free radicals $\mathrm{R}^{*}$ and quickly destroying the inhibitor [Eqs 1 and 2]. After that, the chain reaction has ordinary kinetics.

The situation depends on the order in which the reagents are added.

(1) The inhibitor is added to the substrate before the enzyme. In this case the minimal caffeic acid concentration required for the decrease of the free radical $R$. concentration to the critical value depends only on the initial free radical concentration [ $\left.\mathrm{R}^{*}\right]_{\text {init }}$ in the substrate solution. We suppose that $\mathrm{R}^{*}$ is in equilibrium with the substrate, i.e. $\left[\mathbf{R}^{\prime}\right]_{\text {init }}$ is proportional to substrate concentration. So, the threshold inhibitor concentration is also proportional to the substrate concentration (Fig. 3B, curve 2). In this case, the state of the system (whether the reaction stops or not) is determined before the enzyme addition and depends only on substrate and inhibitor concentrations and not on enzyme concentration (Fig. 3 A, curve 2).

(2) The inhibitor is added to the substrate after the enzyme. The minimal caffeic acid concentration required for the cessation of the reaction depends on the free radical $\mathbf{R}^{\cdot}$ concentration at the moment of inhibitor addition. This concentration of free radical $R^{*}$ is not

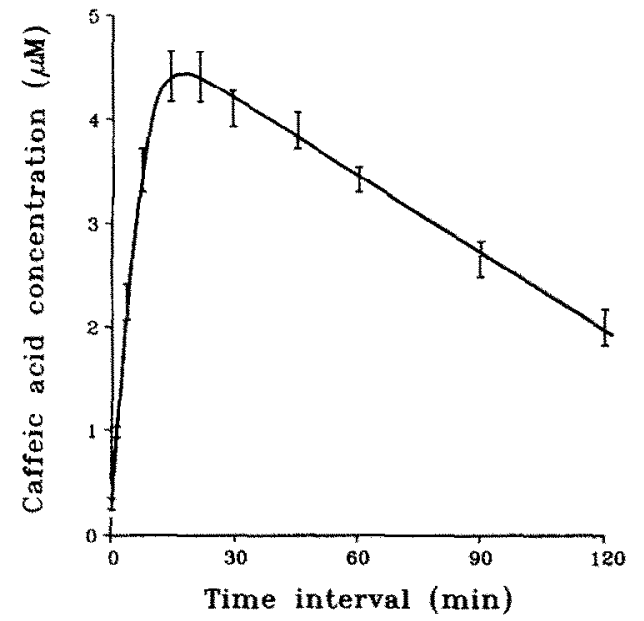

Fig. 4. The dependence of the minimal caffeic acid concentration, required for the cessation of IAA ( $1 \mathrm{mM})$ oxidation catalysed by peroxidase $(0.1 \mu \mathrm{M})$, on the time interval between the reaction initiation and the caffeic acid addition.

equal to $\left[\mathbf{R}^{\prime}\right]_{\text {init }}$. Free radicals $R^{*}$ are formed during the enzymatic, free radical, chain branched reaction of IAA oxidation. Their concentration depends on enzyme and substrate concentrations and on the time interval between the reaction initiation and the inhibitor addition. This agrees with the fact that the threshold inhibitor concentration depends on enzyme and substrate concentrations (Fig. 3, curve 1) and on the moment of the inhibitor addition (Fig. 4).

\section{EXPERIMENTAL}

Horseradish peroxidase $(\mathrm{RZ}=3.0$ ), IAA, caffeic acid, rose bengal and the components of phosphate buffer were obtained from Sigma. Anphen (a new free radical trap) was synthesized and kindly supplied by $\operatorname{Dr}$ A. A. Volod'kin (Institute for Chemical Physics, Russian Academy of Science, Moscow, Russia). Solns were prepared in $0.067 \mathrm{M}$, pH 7.4, $\mathrm{NaPi}$ buffer using $3 \times$ distilled deionized $\mathrm{H}_{2} \mathrm{O}$. Unless otherwise stated, the reaction mixt. contained $0.1 \mu \mathrm{M}$ horseradish peroxidase and $1 \mathrm{mM}$ IAA, the final vol. $3 \mathrm{ml}$.

It is known that peroxidase-catalysed aerobic oxidation of IAA results in the formation of electronically excited species and the appearance of chemiluminescence [8]. The kinetics and spectra of this chemiluminescence are extremely sensitive to the regime of the proceeding reaction [9]. Hence, we used a chemiluminescent method for the study of the peroxidase-catalysed IAA oxidation. Intensity of chemiluminescence was measured with a specially designed chemiluminometer [9]. In addition, we used the spectrophotometric method. The formation of IAA oxidation products was observed by measurement of the difference between $A$ at 242 and $296 \mathrm{~nm}$ (points with identical absorption for the IAA absorption spectrum). The measurements were fulfilled with a 'HITACHI 557' spectrophotometer using the 'two wavelength mode'. All experiments were carried out at $22^{\circ}$. 
Acknowledgement $\mathrm{We}$ are very grateful to $\mathrm{Dr} \mathrm{V}$. V. Lazarev for useful discussions.

\section{REFERENCES}

1. Dörffling, K. (1982) Das Hormonsystem der Pflanzen. Georg Thieme, Stuttgart.

2. Andreae, W. A. (1952) Nature 170, 83.

3. Engelsma, G. (1964) Nature 202, 88.

4. Stonier. T., Yoneda, Y. and Rodriguez-Tormes, F. (1968) Plant Physiol. 43, 1141.

5. Gelinas, D. A. (1973) Plant Physiol. 51, 967.
6. Lee, T. T., Rock, G. L. and Stoessl, A. (1978) Phytochemistry 17, 1721.

7. Haard, N. F. (1978) Z. Pflanzenphysiol. 89, 87.

8. Cilento, G. (1988) Experientia 44, 572.

9. Krylov, S. N., Lazarev, V. V. and Rubin, L. B. (1990) Doklady Biophys. 310-312, 28.

10. Neckers, D. C. (1989) J. Photochem. Photobiol. A: Chem. 47, 1.

11. Foot, C. S. (1991) Photochem. Photobiol. 54, 659.

12. Mottley, C. and Mason, R. P. (1986) J. Biol. Chem. 261, 16860 . 